\title{
Evaluation of Combined Quantification of Cr-Ni Steel using EDS and WDS
}

\author{
Philippe T. Pinard ${ }^{1}$, Ralf Terborg ${ }^{2}$, Tobias Salge ${ }^{3}$ and Silvia Richter ${ }^{1}$ \\ ${ }^{1}$ Central Facility for Electron Microscopy, RWTH Aachen University, Aachen, Germany. \\ ${ }^{2}$ Bruker Nano GmbH, Berlin, Germany \\ ${ }^{3}$ Core Research Laboratories, Natural History Museum, London, UK
}

Classically, wavelength dispersive spectrometers (WDS) are associated with electron microprobe whereas energy dispersive spectroscopy (EDS) is the prominent technique to detect X-rays in scanning electron microscopes (SEM). This distinction is however less clear nowadays with electron microprobes equipped with EDS silicon drift detector and SEMs with WDS. As each spectrometer has advantages and limitations (e.g. resolution, count rate performance, detection efficiency, speed of measurement), there is an obvious advantage to combine both techniques to analyze as quickly and accurately as possible a given sample. The question is therefore which spectrometer should be used to measure which elements and under which analytical conditions.

A series of 15 steel samples from Acerinox, S.A. were selected for this study, as they contain both major ( $\mathrm{Cr}, \mathrm{Fe}$ and $\mathrm{Ni}$ ) and minor elements ( $\mathrm{Si}, \mathrm{Mn}, \mathrm{Co}, \mathrm{Cu}$ and $\mathrm{Mo}$ ) with various concentrations. Measurements were simultaneously performed on a JEOL JXA-8530F microprobe equipped with 5 WDS and a Bruker XFlash EDS (FWHM MnKa $<125 \mathrm{eV}$ ) operated using the Probe for EPMA software. As pointed out by Ritchie et al. [1] the optimal beam current and counting time for EDS and WDS differ. To accommodate both techniques, measurements were performed using an accelerating voltage of $15 \mathrm{kV}$ and beam currents of $8 \mathrm{nA}$ (EDS: $120 \mathrm{~s}$, longest shaping time; WDS: $30 \mathrm{~s}$ peak, $15 \mathrm{~s}$ background) and $80 \mathrm{nA}$ (EDS: $40 \mathrm{~s}$, shortest shaping time; WDS: $10 \mathrm{~s}$ peak, $5 \mathrm{~s}$ background). Measurements were also performed at $6 \mathrm{kV}$ and $20 \mathrm{nA}$, using the $\mathrm{L}$ lines for the transition element metals. In order to apply standard based quantification pure element standards were acquired at $15 \mathrm{kV}$, $8 \mathrm{nA}$. For the $15 \mathrm{kV}, 80 \mathrm{nA}$ samples the $8 \mathrm{nA}$ standards were used.

EDS and WDS had the same accuracy for $\mathrm{Cr}$, Fe and $\mathrm{Ni}(>10 \mathrm{wt} \%$ ), regardless of the analytical conditions used. This was not the case for Mn where EDS results at $80 \mathrm{nA}$ were significantly worse than at $8 \mathrm{nA}$, even for high Mn containing samples (> $5 \mathrm{wt} . \%$ ). Both EDS and WDS experienced problems for samples with low Mn (<1 wt.\%) (Fig. 1). We suspect that the interference of the $\mathrm{Cr} \mathrm{K} \beta$ and, inadequate background positions, in the case of WDS, could explain the larger errors. The quantification of $\mathrm{Si}$ and Mo with concentrations greater than $0.25 \mathrm{wt} . \%$ gave consistent results, although the WDS values were systematically lower (Fig. 2). Potential peak shift between unknowns and standards should be checked. At $80 \mathrm{nA}$, WDS could accurately detect Co and Cu concentrations above 0.1 wt.\%. Note that correcting the interference of the Fe Kß on the Co K $\alpha$ line was required. EDS was unable to correctly measure Co (max. conc. 0.34 wt.\%). Accurate concentrations of Cu were only obtained above 2 wt.\% (Fig. 1). The influence of the background should be investigated. At low beam energy, $6 \mathrm{keV}$, the results were worse for both techniques. As reported in other works [2,3] inaccuracy of the L-line quantification lead to large errors. Similar errors were obtained for both techniques (similar k-ratios). EDS gave slightly better results for major elements, but WDS had the edge for minor elements.

These preliminary results show the complementarity of EDS and WDS to quantify major, minor and trace elements in steel samples. Both techniques have advantages and limitations. Further analysis and improvements will be presented. 
[1] N.W.M. Ritchie et al, Microsc. Microanal. 18 (2012), pp. 892-904.

[2] X. Llovet et al, IOP Conf. Series: Materials Science and Engineering 32 (2012), p. 012014.

[3] Pinard et al, Micros. Microanal. 20 S3 (2014), pp. 700-701.

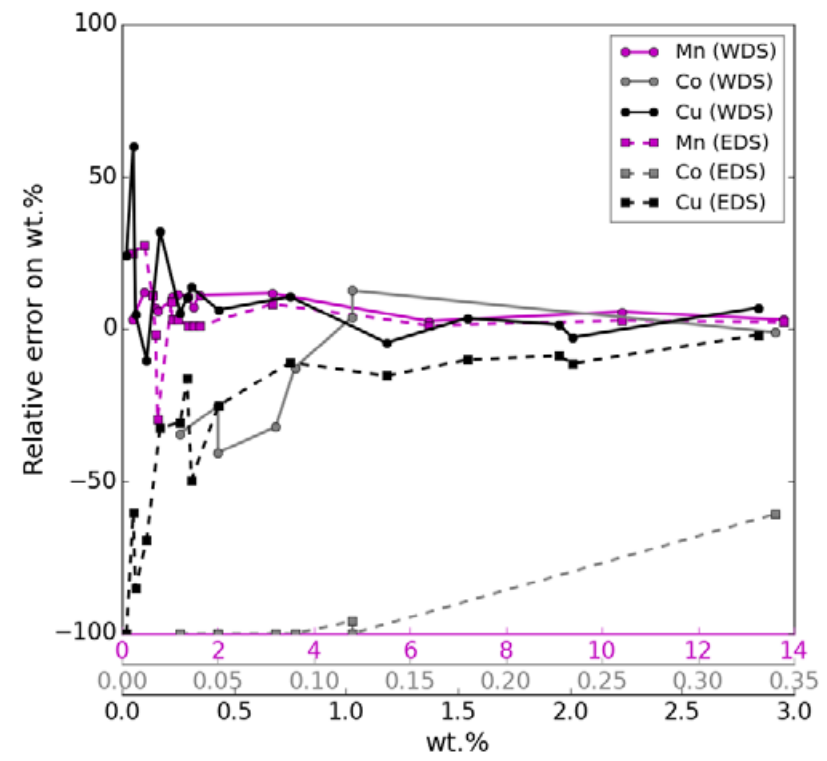

Fig. 1: Relative errors on composition of Mn, Co and $\mathrm{Cu}$ as a function of the nominal concentration from measurements at $15 \mathrm{kV}, 8 \mathrm{nA}$.

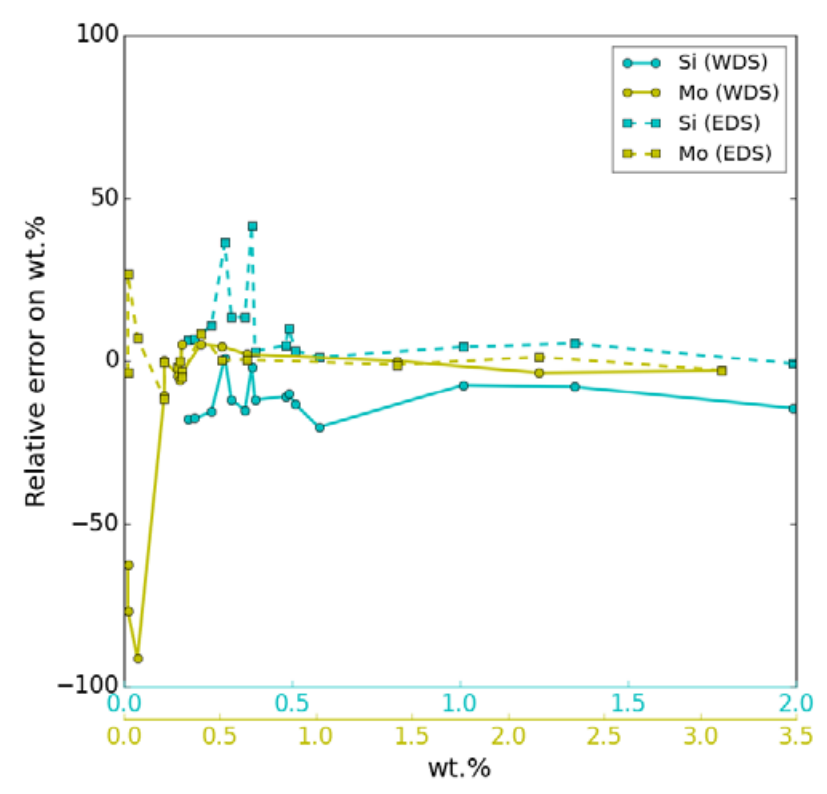

Fig. 2: Relative errors on composition of $\mathrm{Si}$ and Mo as a function of the nominal concentration from measurements at $15 \mathrm{kV}, 8 \mathrm{nA}$.

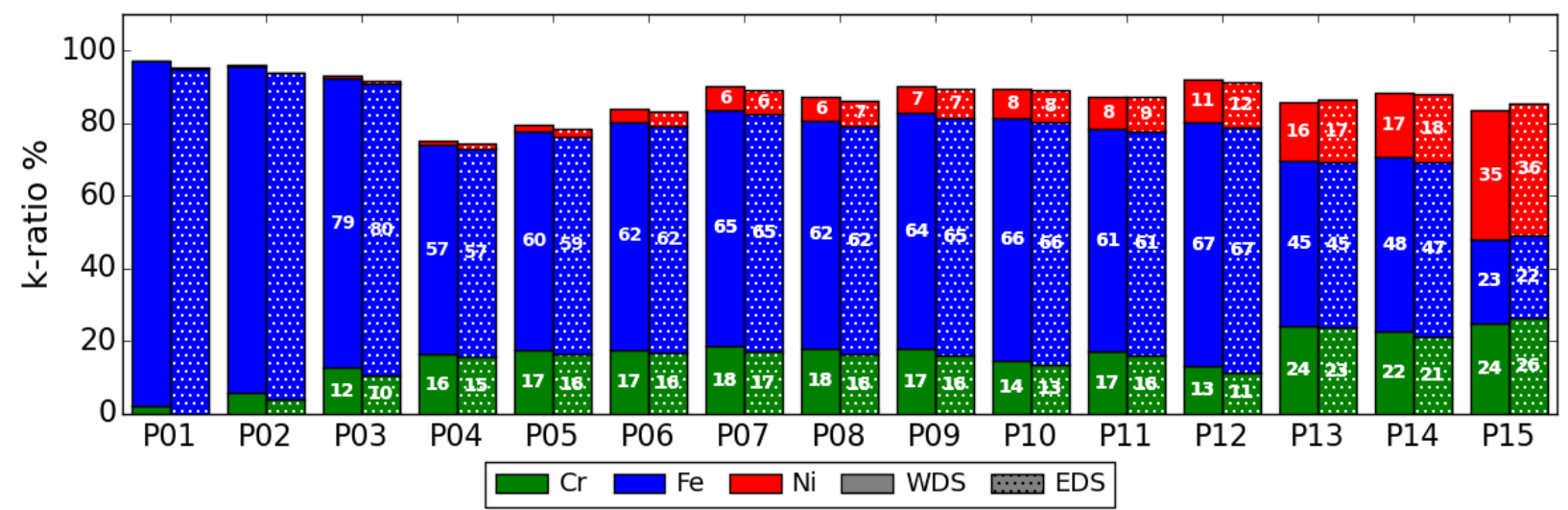

Fig. 3: k-ratios of $\mathrm{Cr}, \mathrm{Fe}$ and $\mathrm{Ni}$ from measurements at 6 kV, $20 \mathrm{nA}$. 